\title{
A POSSIBLE FUNDAMENTAL DIFFERENCE BETWEEN RADIO LOUD AND RADIO QUIET AGN
}

\author{
M. CALVANI \\ Osservatorio Astronomico, Padova, Italia \\ J.W. SULENTIC AND P. MARZIANI \\ University of Alabama, Tuscaloosa, USA \\ D. DULTZIN-HACYAN \\ Instituto de Astronomía, UNAM, México \\ AND \\ M. MOLES \\ Instituto de Astrofisica de Andalucia, Granada, España
}

We report on some striking differences between radio loud and quiet emitters that we found in a comparative analysis of the high and low ionization lines for 52 low redshift AGN (31 loud; 21 quiet).

The broad components of $\operatorname{CIV} \lambda 1549$ and $\mathrm{H} \beta$ were chosen as representative of the high and low ionization lines respectively. Civ $\lambda 1549$ observations were obtained with the Faint Object Spectrograph on the HST. They were retrieved from the HST data archive and matching optical spectra for the region of $\mathrm{H} \beta$ were obtained at several ground based observatories. Details on observations, narrow/broad component deconvolution and profile cleaning from satellite lines (especially FeII) can be found in Marziani et al. (1995). The rest frame for each quasar was determined from the radial velocity of strong narrow lines, typically [OIII] $\lambda \lambda 4959,5007$.

Fig. 1 depicts our results: (1) Radio loud AGN show predominantly redshifted, redward asymmetric $\mathrm{H} \beta$, while Civ $\lambda 1549$ appears broader but predominantly unshifted and symmetric. (2) Radio quiet AGN, on the contrary, show unshifted and symmetric $\mathrm{H} \beta$, and blueshifted and blue-ward asymmetric Civ $\lambda 1549$.

Interesting additional results are: (1) The peak (or 3/4 intensity level) radial velocity of $\mathrm{H} \beta$ almost always exceeds the radial velocity of Crv $\lambda 1549$ in both radio quiet and radio loud AGN. (2) There is evidence that the 


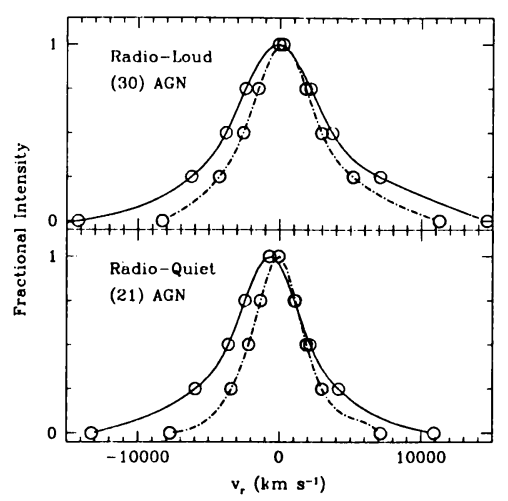

Figure 1. "Median" profiles of $\mathrm{H} \beta$ (dot-dashed line), and CIV $\lambda 1549$ (solid), for the radio loud quasars in our sample (upper panel) and the radio quiet ones (lower panel). The profiles were built interpolating spline functions to the median values of the radial velocity measured at $0,0.25,0.5,0.75,1.0$ peak intensity.

CIv $\lambda 1549$ and $\mathrm{H} \beta$ line profiles are coupled in radio loud $A G N$. Peak shifts of CIv $\lambda 1549$ and $\mathrm{H} \beta$ appear to be correlated. FWHM(CIV $\lambda 1549)$ and FWHM $(\mathrm{H} \beta)$ are also correlated. The ai(1/4) of $\operatorname{Crv} \lambda 1549$ and $H \beta$, even if not correlated show a marked tendency to occupy one quadrant in the diagram ai $(1 / 4)(\mathrm{Crv} \lambda 1549)$ versus ai $(1 / 4)(\mathrm{H} \beta)$. None of those trends is revealed for radio quiet quasars. (3) The luminosity of $\mathrm{H} \beta$ and CIV $\lambda 1549$ are strongly correlated.

A number of properties suggest a relationship between line profiles and radio loudness: (1) Superluminal sources tend to have low $\mathrm{H} \beta$ equivalent width. If we restrict our attention to sources with $\beta_{a p p} \approx 10$, the $\mathrm{H} \beta$ profile is redshifted and redward asymmetric. (2) The dependence of line profile width on the $\mathrm{R}$ (flux of radio core over flux of radio lobe) parameter is the same for both $H \beta$ and CIV $\lambda 1549$. (3) The shift at the line base appears to be correlated with $\mathrm{L}_{\nu, c o r e}$. The largest $\mathrm{L}_{\nu, c o r e}$ is associated with the largest zero intensity. (4) The average radio loud quasar is a less efficient FeII radiator than the average radio quiet quasar. There is some indication, however, that core dominated radio sources may have peculiar FeII emission.

Summing up, our results suggest that: (1) CIv $\lambda 1549$ and $\mathrm{H} \beta$ might well be emitted in the same region in radio loud $A G N$, at variance with radio quiet $A G N$, where several lines of evidence suggest that CIV $\lambda 1549$ is emitted by out-flowing gas, while $\mathrm{H} \beta$ is not. (2) Radio loudness affects the line profiles.

\section{References}

Marziani, P., Sulentic, J.W., Dultzin-Hacyan, D., Calvani, M. and Moles, M. (1995), Ap.J. Suppl., in press. 